\title{
Intraoperative Anwendung von 3-D-C-Bögen
}

\author{
Sven Y. Vetter, Jochen Franke, Paul Alfred Grützner
}

\section{Zusammenfassung}

Seit mehr als einem Jahrzehnt werden 3-D-C-Bögen in unfallchirurgischen Zentren regelmäßig zur Beurteilung von intraoperativen Stellungskontrollen betrieben. Die Verwendung von 3-D-C-Bögen ermöglicht die intraoperative detaillierte Beurteilung von knöchernen anatomischen Regionen, welche mithilfe von konventionellen Bildverstärkern nicht ausreichend dargestellt werden können. Dies betrifft v.a. die intraoperative Darstellung von großen Gelenken sowie des Beckens und der Wirbelsäule. Im Rahmen einer Osteosynthese kann mithilfe der 3-DBildgebung intraoperativ eine unzureichende Reposition der Fraktur oder eine ungünstige Platzierung des Implantats erkannt und umgehend korrigiert werden. Dadurch kann eine Folgeoperation, die bei einer unzureichenden Erstversorgung erforderlich wäre, vermieden werden. Somit stellt die intraoperative 3-D-Bildgebung ein suffizientes Hilfsmittel dar, um die Ergebnisqualität der Frakturversorgung in schwierigen anatomischen Bereichen zu verbessern. Die Durchführung eines intraoperativen 3-D-Scans bedarf eines geschulten OP-Teams sowohl von pflegerischer als auch ärztlicher Seite, sodass eine routinemäßige Anwendung eines 3-D-C-Bogens als sinnvoll erachtet wird.

\section{Intraoperative Application of 3D-C-arms}

Mobile 3D-C-arms have been used routinely for the intraoperative analysis of the reposition and the implant placement in trauma centres for more than a decade. The usage of 3D-C-arms permits the detailed intraoperative evaluation of osseous anatomic regions which can not be displayed in conventional fluoroscopy with a two dimensional C-arm. Especially joints, the pelvis and the spine can sufficiently be illustrated during the surgery with a mobile 3D-C-arm. An insufficient reduction or an intraarticular implant placement can be detected and be corrected immediately avoiding a revision surgery. Mobile 3D-C-arms are a valid tool to improve the quality of fracture care in complex anatomical regions. A routinely application of the mobile 3D-C-arm can be recommended since an experienced team of surgeons and technical assistants is compulsory to ensure a smooth performance of an intraoperative 3Dscan.

zum Goldstandard in der Unfallchirurgie. Diese dienen der 2-dimensionalen Überprüfung des Repositionsergebnisses und der Implantatlage während der Operation. Die Bildqualität der modernen digitalen Bildverstärker wurde in den vergangenen Jahren verbessert, sodass intraoperativ bereits eine Überprüfung des Operationsergebnisses erfolgen kann. Die Beurteilung der Reposition einer Fraktur und der Platzierung des
Implantats kann sich allerdings in einigen anatomischen Regionen mithilfe eines konventionellen Bildverstärkers als schwierig erweisen [1]. Dies trifft v.a. auf die Versorgung von Frakturen und Verletzungen des Beckens, der Wirbelsäule und der großen Gelenke zu. Es ist bekannt, dass die funktionellen Ergebnisse nach einer Fraktur im Gelenkbereich bei einer anatomischen Rekonstruktion der Gelenkfläche besser sind als bei unzureichend reponierten Frakturen [2]. Daher ist das Ziel der operativen Behandlung, eine anatomische Reposition von Frakturen am Becken, der Wirbelsäule sowie im Gelenkbereich zu erzielen [3]. Eine Überprüfung des operativen Ergebnisses ist in einigen anatomischen Regionen mithilfe einer Computertomografie (CT) nach der Operation als Standard anzusehen. Nur wenige Zentren verfügen über ein intraoperatives CT, sodass die Durchführung der CTDiagnostik regelhaft erst im Anschluss an die Operation erfolgt. Dies hat zur Folge, dass mögliche notwendige Korrekturen der Reposition und des Implantats erst in einem Revisionseingriff vorgenommen werden können [4].

3-D-Bildverstärker dienen der intraoperativen Beurteilung des Repositionsergebnisses und der Implantatlage in komplexen anatomischen Regionen.

Um dies zu vermeiden und eine umgehende Korrektur zu ermöglichen, werden zunehmend 3-D-Bildverstärker zur intraoperative Beurteilung von Reposition und Implantatlage verwendet. Außerdem kann meist der intraoperativ angefertigte 3-D-Datensatz das postoperative CT ersetzen und somit die Strahlenbelastung für den Patienten reduzieren. Die Bildqualität der intraoperativen 3-DBildgebung ist im Vergleich zur CT-Diagnostik verringert, lässt allerdings eine ausreichende Beurteilung des Knochens und der Implantate zu [5].
OP-JOURNAL 2014; 30: 144-148

(C) Georg Thieme Verlag KG Stuttgart · New York DOI http://dx.doi.org/10.1055/s-0034-1383303
Die intraoperative Anwendung von konRahmen von operativen Eingriffen am Skelettsystem gehört seit Jahrzehnten 


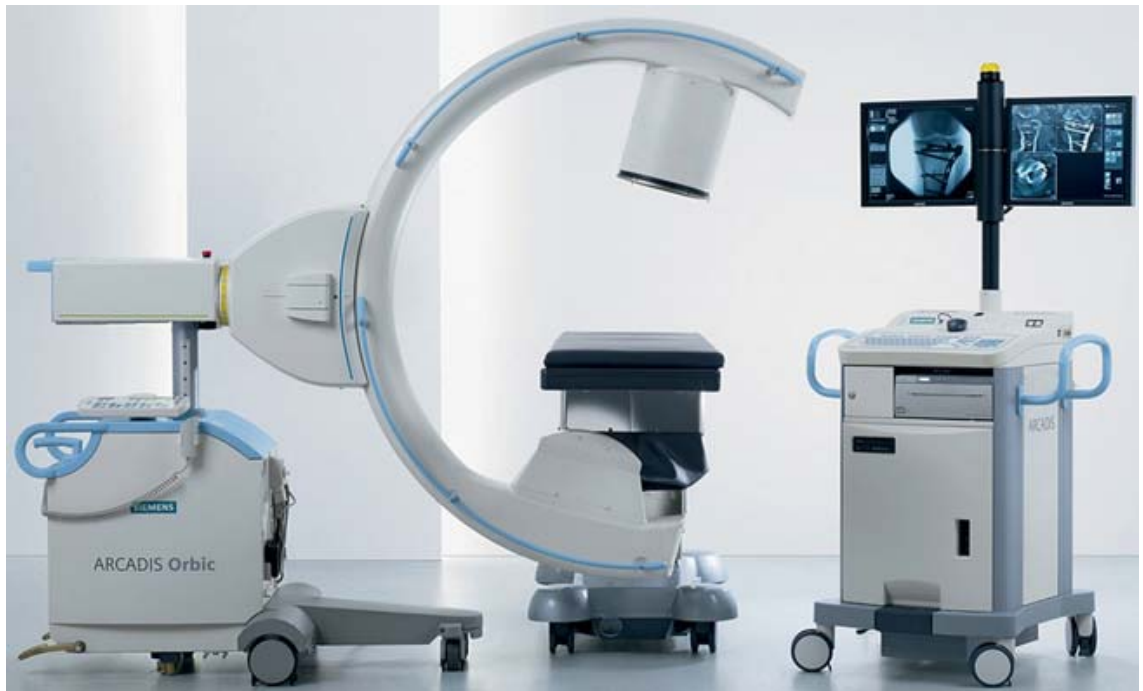

Abb. 1 3-D-C-Bogen zur Durchführung von intraoperativen 3-D-Scans.
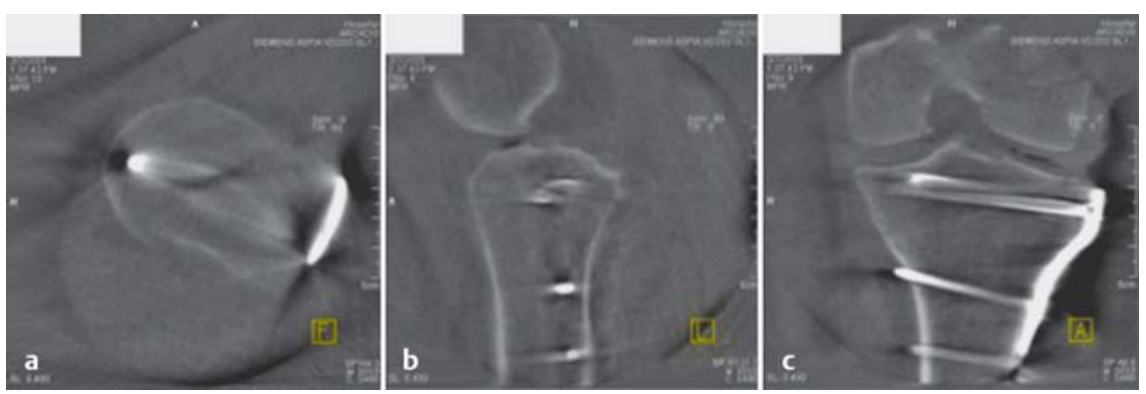

Abb. 2 a bis c Rekonstruktionen aus einem 3-D-Datensatz mit axialen (a), sagittalen (b) und koronaren (c) Schnittebenen.

3-D-C-Bögen ermöglichen eine bessere Beurteilung von Repositionen und Osteosynthesen an Gelenken, dem Becken und der Wirbelsäule als konventionelle 2-D-C-Bögen.

In mehreren Studien konnte gezeigt werden, dass durch eine intraoperative 3-D-Bildgebung eine nicht unerhebliche Anzahl (20-30\%) an korrekturbedürftigen Befunden festgestellt wurde, welche eine umgehende intraoperative Korrektur nach sich zog [6-8].

Durch die Anwendung von intraoperativer 3-D-Bildgebgung können korrekturbedürftige Befunde dargestellt werden, die umgehend korrigiert werden können.

Im Rahmen einer Untersuchung der operativen Versorgung von Fersenbeinfrakturen mit Gelenkbeteiligung konnte demonstriert werden, dass eine adäquate Darstellung der hinteren Gelenkfläche des unteren Sprunggelenks in der konventionellen Durchleuchtung nur eingeschränkt möglich war, sodass ungenügende Repositionen oder inadäquate
Schraubenlagen nicht erkannt werden konnten. Dies hatte zur Folge, dass die Revisionsrate von korrekturbedürftigen Befunden am Fersenbein, welche mithilfe einer intraoperativen 3-D-Bildgebung detektiert wurde, bei über $20 \%$ lag [8]. Ähnliche Ergebnisse wurden bei operativen Eingriffen am oberen Sprunggelenk beobachtet. Untersuchungen von Osteosynthesen am oberen Sprunggelenk zeigten in 20 bzw. 34\% korrekturbedürftige Befunde nach konventioneller BVDarstellung [9]. Auch diese wurden erst nach einer 3-D-Darstellung erkannt und konnten korrigiert werden. Auswertungen von Daten aus unserem Haus zeigen analoge Ergebnisse. Bei Operationen an Wirbelsäule/Becken und an großen Gelenken konnte in nahezu 30\% der Fälle die intraoperative 3-D-Bildgebung einen korrekturbedürftigen Befund feststellen, welcher mit einem konventionellen BV nicht darstellbar war. Die intraoperative Anwendung von 3-D-C-Bögen zur 3-dimensionalen Beurteilung des Frakturausmaßes, der Reposition und der Implantatlage erfolgt routinemäßig in unfallchirurgischen Zentren bereits seit mehr als 10 Jahren. Die intraoperative 3-D-Bildgebung als modernes bildgebendes Verfahren in der Unfallchirurgie hat sich im OP etabliert und ist ein fester Bestandteil des täglichen Betriebs im OP [10].

\section{Technische Voraussetzungen}

Ein mobiler 3-D-Bildverstärker unterscheidet sich von konventionellen 2-DGeräten in seinem Aufbau. Im Gegensatz zu herkömmlichen Bildverstärkern bleibt der Zentralstrahl eines 3-D-BV zwischen der Strahlenquelle und dem Detektor bei Rotation des C-Bogens immer auf das Isozentrum fokussiert [11]. Dies ermöglicht, dass bei Rotation des Bildverstärkers sämtliche Projektionen im Drehzentrum des Bildverstärkers verbleiben. Während einer kontinuierlichen Rotation um $190^{\circ}$ in festgelegten Winkelabständen wird eine definierte Anzahl - meistens 100 - von digitalen 2-DAufnahmen erstellt (Abb. 1). Aus diesen isozentrischen Projektionsaufnahmen der Region werden über einen speziellen Algorithmus 3-D-Bilder in einem Datenwürfel mit einer Kantenlänge von 12,5 cm erzeugt (Abb. $\mathbf{2}$ ).

An einem angeschlossenen Monitor werden die Aufnahmen nach Beendigung der Rotationsbewegung visualisiert und können vom Operateur betrachtet und beurteilt werden. Die Betrachtungsebenen sind am Monitor per Hand einzustellen. Dabei haben sich die Einstellungsebenen aus der CT-Diagnostik bewährt, um eine standardisierte, gewohnte Betrachtungsweise zu erhalten. Dadurch können die intraoperativen Bilder auch mit präoperativen CT-Bildern abgeglichen werden.

Auf Wunsch des Operateurs können zusätzlichen Ebenen frei eingestellt werden, um auf spezielle Fragestellungen wie Repositionen von Fragmenten und Schraubenpositionierungen einzugehen. Dadurch können Schrauben einzeln eingestellt sowie deren Verlauf und Lage beurteilt werden (Abb. 3 ).

Die Qualität des Bildmaterials ist zum einen von der eingebrachten Menge von Implantaten und zum anderen von den sich im Strahlengang befindlichen Weichteilen abhängig. Artefakte können die Bildbeurteilung erheblich einschränken, sodass auf die Lagerung des Patienten während der Durchleuchtung und auf die Platzierung des Implantats geachtet werden sollte. 


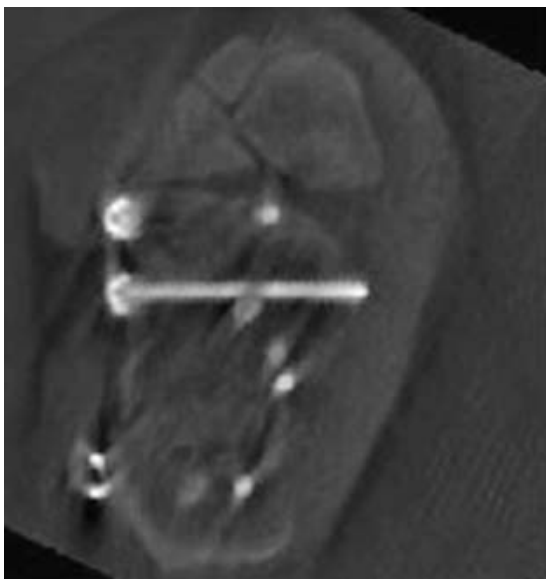

Abb. 3 Darstellung einer Schraube in voller Länge zur Beurteilung der Lage.

Artefakte können die intraoperative Bildbeurteilung einschränken. Durch eine regelrechte Lagerung und Einstellung des 3-D-Bogens können diese reduziert werden.

Dadurch können Überlagerungen z.B. von gesunden Extremitäten, die im Zentralstrahl positioniert sind, vermieden werden. Außerdem sollte bei der Lagerung auf einen röntgendurchlässigen OP-Tisch im Bereich des Strahlenfelds geachtet werden. Dies wird durch Carbontische oder Carbon-Anbauteile ermöglicht.

\section{Durchführung eines intraoperativen 3-D-Scans}

Die Anwendung eines 3-D-Scans erfolgt nach den Vorgaben des Operateurs. Der Zeitpunkt einer 3-D-Bildgebung wird typischerweise nach einer konventionellen Kontrolle der Reposition und Osteosynthese gewählt. Die Indikation der intraoperativen Kontrolle ist die Überprüfung der Reposition der Fraktur und die Beurteilung der Implantatlage. Die Durchführung eines intraoperativen 3-D-Scans kann auch dann gerechtfertigt sein, wenn noch keine endgültige Osteosynthese erfolgte, sondern lediglich die Überprüfung der Lage von Führungsdrähten im Bereich von komplexen anatomischen Regionen wie Wirbelsäule und Becken erforderlich ist.

Die Anfertigung eines 3-D-Scans kann auch bereits vor einer Operation sinnvoll sein, sofern keine ausreichende präoperative Diagnostik erfolgte.

Die Durchführung eines 3-D-Scans erfordert vom Operationsteam Routine im Umgang mit dem System und bedarf

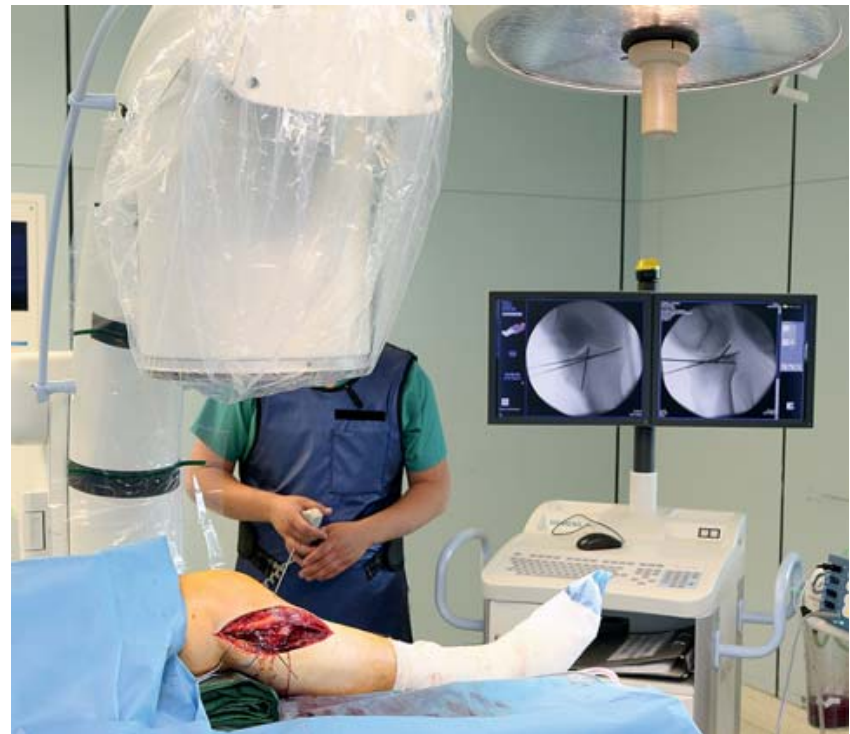

Abb. 4 Darstellung der Region zur Beurteilung der Größe des Bildausschnitts in 2 Ebenen.
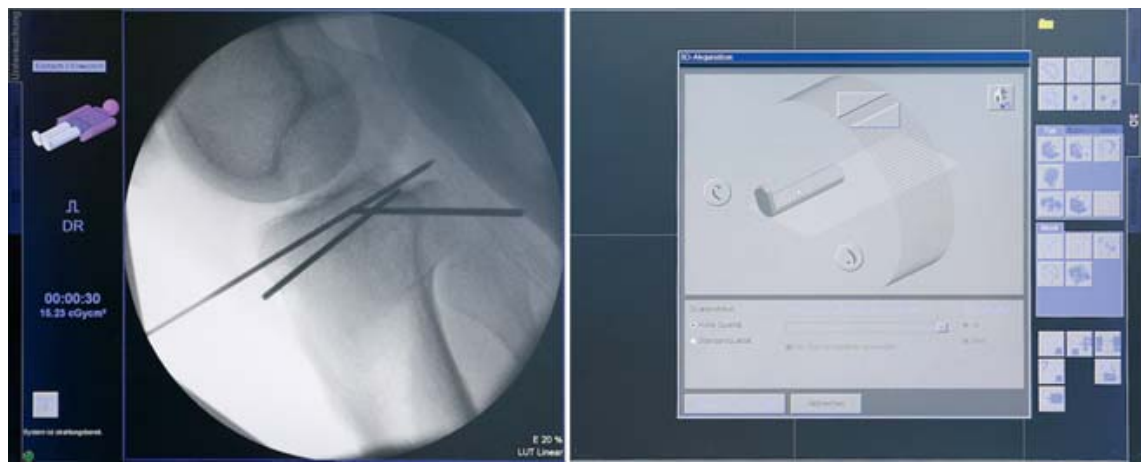

Abb. 5 Einstellen der Position des 3-D-C-Bogens und der Lage des Patienten.

mehr Zeit als eine konventionelle Kontrolle mit einem 2-D-Bildverstärker.

Der 3-D-Bildverstärker muss zunächst exakt positioniert werden, um den richtigen Bildausschnitt abzubilden. Dabei wird der 3-D-Bildverstärker analog zu einem 2-D-Gerät zum Patienten ausgerichtet. Die Position wird sowohl a.-p. als auch im seitlichen Strahlengang überprüft (Abb.4). Im Anschluss wird der Modus der Durchleuchtung ausgewählt und die Lage des Patienten zum Bildverstärker festgelegt (Abb.5), bevor sich der Durchleuchtungsvorgang mit 100 digitalen Bildern anschließt (Abb. 6). Die Auswertung des Bilddatensatzes kann erst nach der digitalen Berechnung erfolgen, die ca. 15 Sekunden in Anspruch nimmt. Nach Erstellung des Bilddatensatzes schließt sich die Bearbeitung der Bilder mit Festlegung der Betrachtungsebenen durch den Operateur an (Abb. 7).

Die Durchführung einer intraoperativen 3-D-Bildgebung an den Extremitäten weist meistens die geringsten Schwierigkeiten auf. Regelhaft wird diese - wie zuvor beschrieben - nach der Reposition und Osteosynthese vorgenommen, nachdem bereits eine konventionelle BVKontrolle in 2 Ebenen erfolgte. Dabei wird die Extremität so gelagert, dass die Region, die zu beurteilen ist, im Zentrum des Strahlengangs in beiden Ebenen liegt. Dies ist vor dem 3-D-Scan gewissenhaft zu prüfen, um den richtigen Bildausschnitt darzustellen. Zudem ist bei der Lagerung auf eine stabile Positionierung der Extremität zu achten, da es durch ein akzidentielles Verändern der Position zu Bewegungsartefakten kommen kann, welche eine Beurteilung des Datensatzes erschweren. Im Anschluss ist ein Kollisionstest durchzuführen, bei dem der C-Bogen um $190^{\circ}$ gedreht wird. Nach Festlegung der Position des Patienten, der Lagerung und der Stellung des Bildverstärkers kann der Scanvorgang gestartet werden. Dabei ist ein ausreichender Abstand des OP-Teams zur Strahlenquelle zu beachten, um eine unnötig hohe Strahlenexposition der Mit- 


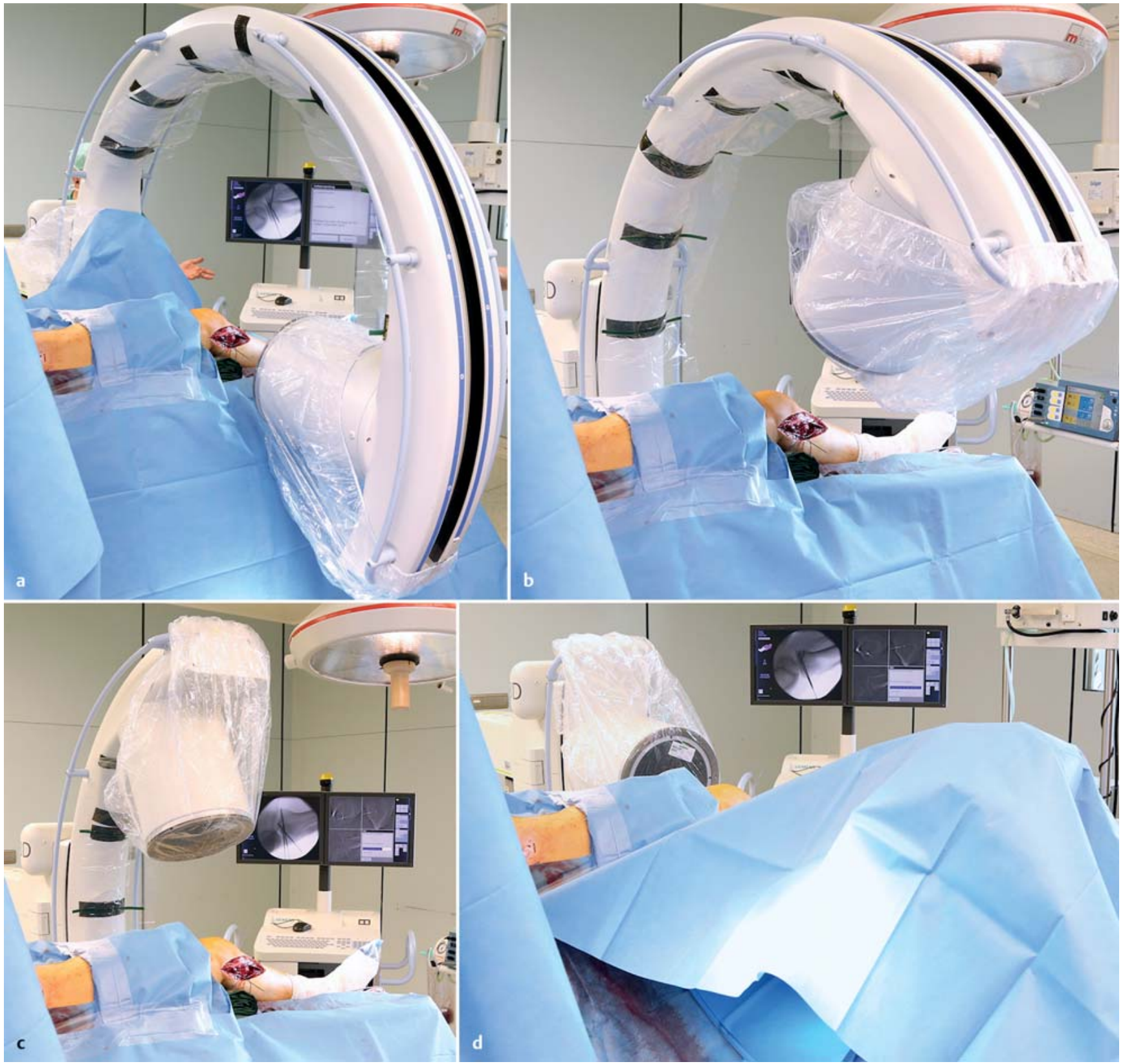

Abb. 6 a bis d Durchführung eines 3-D-Scans mit 100 digitalen Bildern.

arbeiter zu vermeiden. Die Bedienung des Bildverstärkers mit Durchführung des Scans wird analog zu den 2-D-Aufnahmen durch das nicht sterile OP-Personal vorgenommen. Das Vorgehen bei intraoperativen 3-D-Scans der Wirbelsäule und des Beckens ist analog. Bei der Lagerung des Patienten ist präoperativ darauf zu achten, dass die Durchführung des Scan der betroffenen Region möglich ist und nicht durch Hindernisse unmöglich gemacht wird. Zudem ist v.a. im Bereich des Beckens auf die Größe des Bildausschnitts zu achten, welcher bei einer Kantenlänge von $12,5 \mathrm{~cm}$ nur einen begrenzten Bereich der Region abbildet.

\section{Auswertung}

Die Auswertung des Bilddatensatzes erfolgt intraoperativ durch den Operateur. Dieser stellt die Betrachtungsebenen analog zu den standardisierten Rekonstruktionsebenen einer Computertomografie direkt am Monitor ein. Nach Einstellung der Ebenen kann die Analyse der Reposition und der Positionierung des Implantats erfolgen. Das Hauptaugenmerk wird hierbei auf die anatomische Rekonstruktion der Gelenkfläche und auf die korrekte Implantatplatzierung gelegt. Sollten Stufen, Spalten bzw. knöcherne Defekte in der Gelenkfläche sowie intraartikuläre Schrauben detek- tiert werden, ist wenn möglich eine intraoperative Korrektur vorzunehmen und das Vorgehen einer 2-D- mit anschließender 3-D-Kontrolle zu wiederholen. Sämtliche intraoperativ angefertigten Datensätze werden vom Operateur dokumentiert und über eine Anbindung an das klinikinterne Krankenhausinformationssystem archiviert. Eine Codierung der Prozedur ist durch die OPS-Ziffern 3-20x sowie 3-996 und 3-990 möglich. Die Verschlüsselung dieser Codes ist allerdings zum aktuellen Zeitpunkt nicht erlösrelevant. 


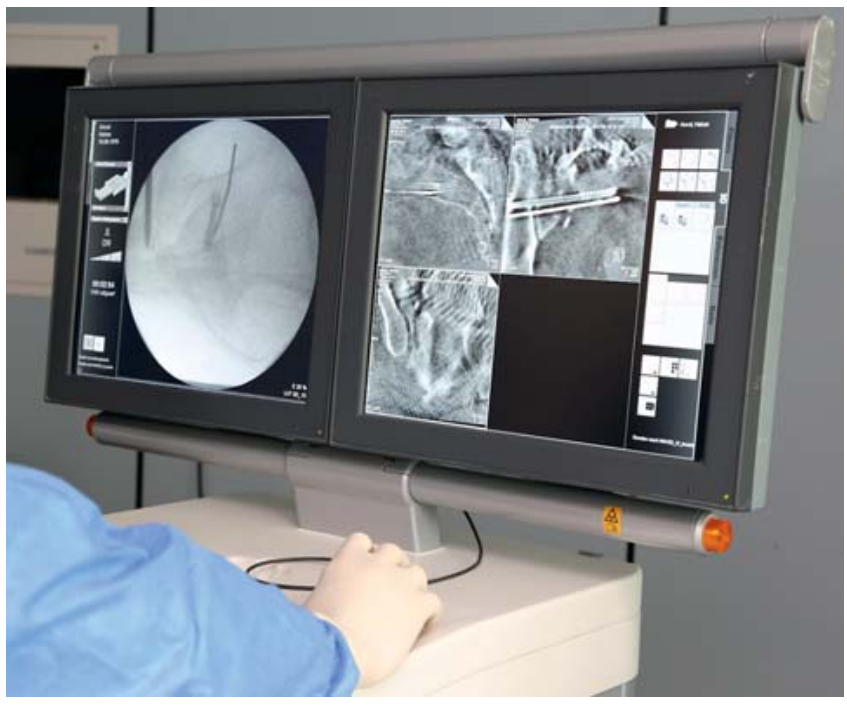

Abb. 7 Bearbeitung des Datensatzes durch den Operateur und Einstellung der Standardebenen.

\section{Fazit}

Für die intraoperative Beurteilung der Frakturreposition und der Implantatpositionierung spielt die Bildgebung mit 3-D-C-Bögen v.a. in komplexen anatomischen Regionen eine wichtige Rolle.

Trotz gewissenhaftem operativem Vorgehen und Kontrollen mit konventionellen C-Bögen zeigen Studien eine intraoperative Korrekturrate von $20-30 \%$ unzureichender Repositionen oder Implantatlagen, die durch eine 3-D-Bildgebung detektiert wurden.

Eine umgehende Korrektur der Reposition kann im Rahmen der Operation erfolgen und Folgeoperationen können dadurch vermieden werden. Eine regelmäßige Anwendung durch ein geschultes Operationsteam wird für einen reibungslosen Ablauf des Scanvorgangs empfohlen.

\section{Literatur}

${ }^{1}$ Atesok K, Finkelstein J, Khoury A et al. The use of intraoperative three-dimensional imaging (ISO-C-3D) in fixation of intraarticular fractures. Injury 2007; 38: 1163-1169

2 Franke J, von Recum J, Suda AJ et al. Intraoperative three-dimensional imaging in the treatment of acute unstable syndesmotic injuries. J Bone Joint Surg Am 2012; 94: 1386-1390

3 Kendoff D, Citak M, Gardner MJ et al. Intraoperative 3D imaging: value and consequences in 248 cases. J Orthop Trauma 2009; 66: 232-238

${ }^{4}$ Grutzner PA, Beutler T, Wendl K et al. Navigation an der Brust- und Lendenwirbelsäule mit dem 3D- Bildwandler. Chirurg 2004; 75: 967-975

${ }^{5}$ Konig B, Erdmenger U, Schroder RJ et al. Evaluation der Bildqualität des Iso-C3D-Bildwandlers im Vergleich mit dem CT. Unfallchirurg 2005; 108: 378-385

${ }^{6}$ Kendoff D, Ortega G, Citak M et al. Limitations and pitfalls of 3-D fluoroscopic navigation in orthopaedic trauma surgery. Technol Health Care 2009; 17: 133-140

7 Richter M, Zech S. Intraoperative 3-dimensional imaging in foot and ankle trauma-experience with a second-generation device
(ARCADIS-3D). J Orthop Trauma 2009; 23: 213-220

${ }^{8}$ Rübberdt A, Feil R, Stengel D et al. Die klinische Wertigkeit des ISO-C3D bei der Osteosynthese des Fersenbeins. Unfallchirurg 2006; 109: 112-118

${ }^{9}$ Richter M, Geerling J, Zech S et al. Intraoperative three-dimensional imaging with a motorized mobile C-arm (SIREMOBIL ISO-C-3D) in foot and ankle trauma care: a preliminary report J Orthop Trauma 2005: 19: 259-266

${ }^{10}$ Franke J, von Recum J, Wendl K et al. Intraoperative dreidimensionale Bildgebung - nützlich oder notwendig? Unfallchirurg 2013; 116: 185-190

11 Rock C, Linsenmaier U, Brandl R et al. Vorstellung eines neuen mobilen C-Bogen-/CTKombinationsgerät (ISO-C-3D) Erste Ergebnisse der 3D-Schnittbildgebung. Unfallchirurg 2001; 104: 827-833

\section{Dr. Sven Y. Vetter, MBA}

Facharzt für Orthopädie und

Unfallchirurgie, Spez. Unfallchirurgie

Dr. Jochen Franke

Oberarzt der Klinik für Unfallchirurgie und Orthopädie

Prof. Dr. Paul Alfred Grützner

Direktor der Klinik für Unfallchirurgie und Orthopädie

MINTOS - Medical Imaging

and Navigation in Trauma

and Orthopaedic Surgery

BG Unfallklinik Ludwigshafen

Ludwig-Guttmann-Straße 13

67071 Ludwigshafen

Tel.: 0621/68100

Fax: 0621/68102986

sven.vetter@bgu-ludwigshafen.de aed@bgu-ludwigshafen.de jochen.franke@bgu-ludwigshafen.de 